EPJ Web of Conferences 116, 11012 (2016)

DOI: $10.1051 /$ epjconf/201611611012

(C) Owned by the authors, published by EDP Sciences, 2016

\title{
(Very)-high-energy gamma-ray astrophysics: The future
}

\author{
Alessandro De Angelis ${ }^{\mathrm{a}}$ \\ INFN Padova, via Marzolo 8, 35141 Padova, Italy; LIP/IST, Av. Elias Garcia 14, \\ 1000 Lisboa, Portugal
}

\begin{abstract}
Several projects planned or proposed can significantly expand our knowledge of the high-energy Universe in gamma rays. Construction of the Cherenkov telescope array CTA is started, and other detectors are planned which will use the reconstruction of extensive air showers. This report explores the near future, and possible evolutions in a longer term.
\end{abstract}

\section{Introduction}

Among cosmic rays, photons are particularly important. Being neutral they can travel long distances without being deflected by galactic and extragalactic magnetic fields, hence they allow us to directly study their emission sources. The gamma emission is also related to the emission of charged cosmic rays, and to the emission of neutrinos. Finally, gamma-rays can be the signature of new physics at fundamental scales, new particles in particular. These facts have pushed the scientific community during the last years to study high-energy gamma rays; many new results have been obtained since the beginning of the millennium, in particular thanks to the imaging atmospheric Cherenkov telescopes (IACTs) H.E.S.S., MAGIC and VERITAS. See [1] for a summary of the main achievements.

Still many problems are however open, in particular:

- The study of the origin of cosmic rays. Thanks to the first generation of IACTs and to the AGILE and Fermi satellites, it is well established nowadays that supernova remnants (SNRs) can accelerate cosmic rays up to some $100 \mathrm{TeV}$. The mechanism of acceleration of particles up to the $\mathrm{PeV}$ is still to be established. Above some PeV, it is common belief that the acceleration sites should be extragalactic, but the mechanism, possibly related to the behavior of matter near supermassive black holes (SMBH), is still to be understood.

- The study of the propagation of extragalactic gamma rays, which is a probe of cosmology and of the interaction of gamma rays with the cosmic background.

Once produced, photons must travel towards the observer. Electron-positron $\left(e^{-} e^{+}\right)$pair production in the interaction of VHE photons off extragalactic background photons is the main source of opacity of the Universe to $\gamma$-rays whenever the corresponding photon mean free path

\footnotetext{
a e-mail: alessandro.deangelis@pd.infn.it
}

This is an Open Access article distributed under the terms of the Creative Commons Attribution License 4.0, which permits unrestricted use, distribution, and reproduction in any medium, provided the original work is properly cited. 

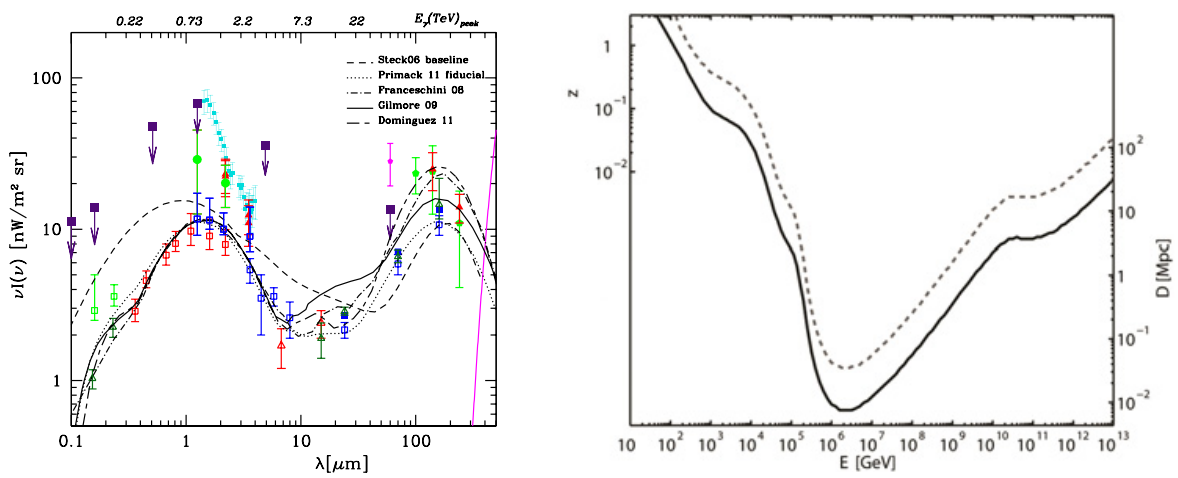

Figure 1. Left: Spectral energy distribution of the EBL as a function of the wavelength. Open symbols correspond to lower limits from galaxy counts while filled symbols correspond to direct estimates. The curves show a sample of different recent EBL models, as labeled. On the upper axis the TeV energy corresponding to the peak of the $\gamma \gamma$ cross section is plotted. From L. Costamante, IJMPD 22 (2013) 1330025. Right: Curves corresponding to the so-called gamma-ray horizon $\tau(E, z)=1$ (lower) and to a survival probability of $e^{-\tau(E, z)}=1 \%$ (upper). Adapted from A. de Angelis, G. Galanti, M. Roncadelli, MNRAS 432 (2013) 3245.

is of the order of the source distance, or smaller. The cross-section is maximized $\left(\sigma_{\max } \simeq\right.$ $1.7 \times 10^{-25} \mathrm{~cm}^{2}$ ) for background photons of energy:

$$
\epsilon(E) \simeq\left(\frac{900 \mathrm{GeV}}{E}\right) \mathrm{eV} .
$$

The maximum photon density corresponds to the CMB; its total density is about 400 photons per cubic centimeter.

A region of particular interest is the so-called extragalactic background light (EBL), i.e., the light in the visible and near infrared regions. It consists of the sum of starlight emitted by galaxies throughout their whole cosmic history, plus possible additional contributions, like, e.g., light from hypothetical first stars that formed before galaxies were assembled. Therefore, in principle the EBL contains important information about both the evolution of baryonic components of galaxies and the structure of the Universe in the pre-galactic era. Note that the light is redshifted by a factor $(1+z)$ due to the expansion of the Universe, and thus, the visible light from old sources is detected as infrared. The density of EBL photons in the region near the visible can be derived by direct deep-field observations, and by constraints on the propagation of VHE photons. A plot of the present knowledge on the density of photons in the EBL region is shown in Fig. 1, left. The corresponding opacity of the Universe is illustrated in Fig. 1, right.

- The examination of the ultimate nature of matter and of physics beyond the Standard Model, in particular through searches for dark matter, for new particles in general in particular by the determination of the energy density of the vacuum, for the effects of quantum gravity. High-energy gamma astrophysics is sensitive to energy scales important for particle physics, in particular the $100 \mathrm{GeV}$ scale expected for cold dark matter; the TeV scale, where supersymmetric particles might appear; and finally, it might be possible to access indirectly the unification scale and the Planck scale: an energy $\sim 10^{19} \mathrm{GeV}$, corresponding to a mass $\sqrt{\hbar c / G}$.

We shall define in the following as high-energy (HE) photons the photons above $30 \mathrm{MeV}$; as very-highenergy (VHE) the photons above $30 \mathrm{GeV}$. 

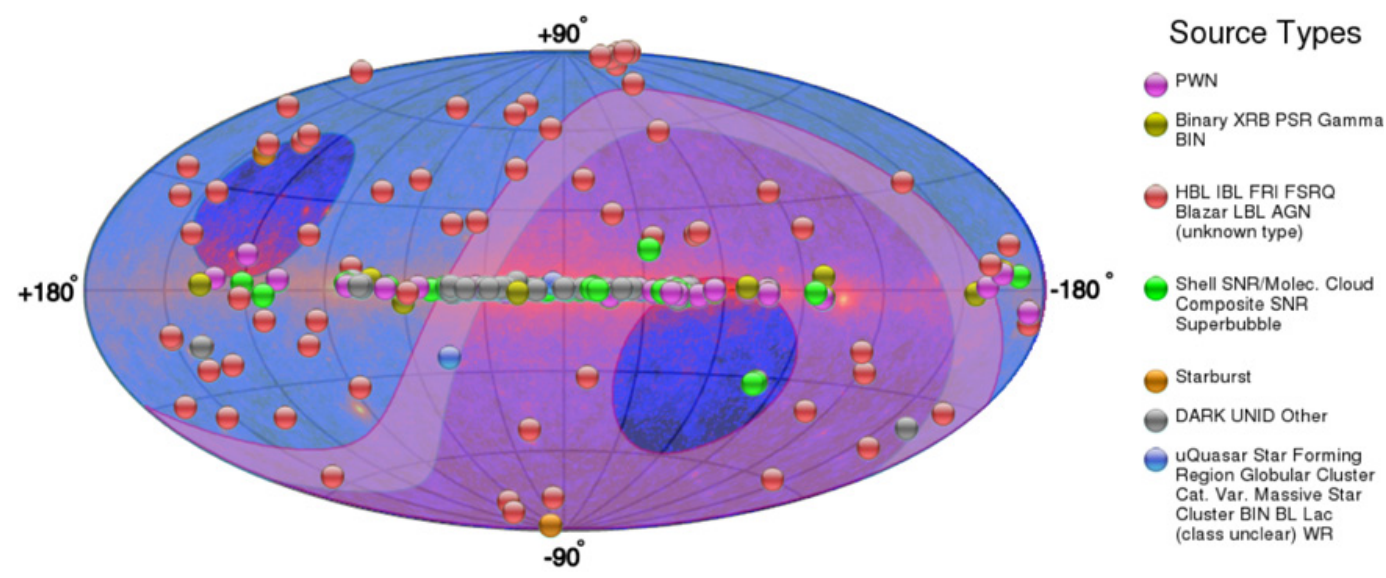

Figure 2. Sources of VHE emission plotted in galactic coordinates. The background represents the highenergy gamma-rays detected by Fermi-LAT. The clear blue and pink regions correspond respectively to the visible regions within $30^{\circ}$ of the zenith from a detector at the tropic in the Northern hemisphere (approximately like MAGIC or VERITAS) and at the Southern hemisphere (approximately like H.E.S.S.). From http://tevcat.uchicago.edu/, January 2016.

A look to the sources of cosmic gamma-rays in the HE region shows a diffuse background, plus a set of localized emitters. Roughly 3000 HE emitters have been identified up to now, mostly by the Fermi-LAT, and some 200 of them are VHE emitters as well (Fig. 2).

About half of the VHE gamma-ray emitters are objects in our Galaxy; most of them can be associated to supernova remnants (SNR). The remaining half are extragalactic, and the space resolution of present detectors (slightly better than $0.1^{\circ}$ ) is not good enough to associate them with particular points in the host galaxies; we believe, however, that they are produced in the vicinity of supermassive black holes in the galaxies. The abundance of galactic objects can be explained by the fact that, being closer, they suffer a smaller attenuation.

The sky exhibits in particular transient events from steady emitters ("flares") and burst of gammarays from previously dark regions ("gamma-ray bursts").

\section{How to detect photons at different energies: Present and near future}

Due to the conversion probability in the atmosphere (whose thickness is about 28 radiation length at sea level) only satellite-based detectors can detect primary X/ $\gamma$-rays. Satellites are anyway small, being of about $1 \mathrm{~m}^{2}$ their maximum dimensions because of the cost of space technology. If the energy of the primary particle is large enough, some of the products of the shower generated by the interaction with the atmosphere, acting as a calorimeter, can reach ground-based detectors. It is appropriate to build such detectors at high altitudes, where atmospheric dimming is lower. The area sampled by ground-based detectors can be much larger than the typical areas of satellites. Since the fluxes of high-energy photons are low and decrease rapidly with increasing energy, $\mathrm{TeV}$ and $\mathrm{PeV}$ gammas can be detected only from the atmospheric showers they produce, i.e., by means of ground-based detectors.

\subsection{Satellites}

Satellite gamma telescopes can detect the primary photons at energies lower than ground-based telescopes. 
Main figures of merit for a satellite-borne detector are its effective area (i.e., the product of the area times the detection efficiency), the energy resolution, the space or angular resolution (called as well point-spread function, or PSF).

Detectors on satellites have a small effective area, of order of $1 \mathrm{~m}^{2}$ maximum, which limits the sensitivity. They have a large duty cycle, and they suffer a low rate of background events, since they can be coupled to anticoincidence systems rejecting the charged cosmic rays. They have a large cost, dominated by the costs of launch and by the strong requirements of instruments which must be sent in space, with little or no possibility of intervention to fix possible bugs.

The Fermi satellite, in orbit since 2008, has an effective area of about $1 \mathrm{~m}^{2}$; its function is double, since it can also operate as a trigger for ground-based telescopes.

\subsection{Ground-based gamma detectors}

Ground-based VHE telescopes such as HAWC, H.E.S.S., MAGIC and VERITAS, detect the atmospheric showers produced by primary photons and cosmic rays of energy higher than those observed by satellites.

The two kinds of detectors (on satellite and at ground) are complementary. At energies below $1 \mathrm{GeV}$ or so, the showers generated by photons do not have the time to develop properly, and thus the only way to detect such photons below this energy is related to the use of satellites. Ground-based detectors have a huge effective area, so their sensitivity is high; they detect a huge amount of background events, but they have low cost.

There are two main classes of ground based VHE gamma detectors: the EAS arrays and the Cherenkov telescopes.

\subsubsection{EAS detectors}

The EAS detectors, such as HAWC which is presently in operation, are large arrays of detectors sensitive to charged secondary particles generated in the atmospheric showers. They have a high duty cycle and a large field-of-view, but a relatively low sensitivity. The energy threshold of such detectors is rather large, in the order of a few hundred $\mathrm{GeV}$ - a shower initiated by a $1 \mathrm{TeV}$ photon typically has its maximum $8 \mathrm{~km}$ a.s.l.

The principle of operation is the same as the one for the UHE cosmic rays detectors like Auger, i.e., direct sampling of the charged particles in the shower. This can be achieved:

- either using a sparse array of scintillator-based detectors, Resistive-Plate Chambers (RPC), or water Cherenkov pools;

- or by effective covering of the ground, to ensure efficient collection and hence lower the energy threshold.

Possibly a combination of the techniques above can be used, with a central region more dense, to allow discrimination between hadron-generated and photon-generated showers, and a peripheral region.

The energy threshold of EAS detectors is at best in the $0.5 \mathrm{TeV}-1 \mathrm{TeV}$ range, so they are built to detect UHE photons as well as the most energetic VHE gammas. At such energies fluxes are small and large surfaces of order of $10^{4} \mathrm{~m}^{2}$ are required.

Concerning the discrimination from the charged cosmic ray background, muon detectors devoted to hadron rejection may be present. Otherwise, it is based on the reconstructed shower shape. The direction of the detected primary particles is computed from the arrival times with an angular precision of about $1^{\circ}$. The calibration can be performed by studying the shadow in the reconstructed directions caused by the Moon. Energy resolution is poor. 

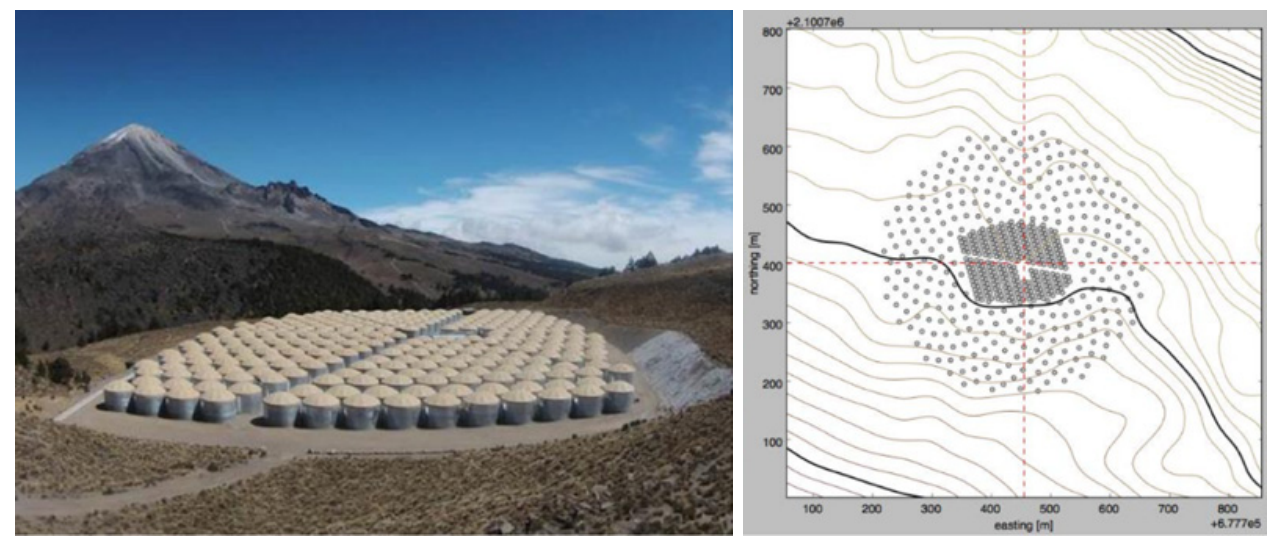

Figure 3. Left: the HAWC detector. Right: its possible extension.

Somehow, the past generation EAS detectors were not sensitive enough and just detected a handful of sources. This lesson lead to a new EAS observatory with much larger sensitivity: the High Altitude Water Cherenkov detector HAWC, inaugurated in 2015.

HAWC (Fig. 3) is a very high-energy gamma-ray observatory located in Mexico at about $4100 \mathrm{~m}$ asl and latitude $19^{\circ} \mathrm{N}$. It consists of 300 steel tanks of $7.3 \mathrm{~m}$ diameter and $4.5 \mathrm{~m}$ deep, covering an instrumented area of about $22000 \mathrm{~m}^{2}$. Each tank is filled with purified water and contains four PMT of 3.8 inch diameter and one high-quantum efficiency 10-inch PMT, which observe the Cherenkov light emitted in water by superluminal particles in atmospheric air showers. Photons traveling through the water typically undergo Compton scattering or produce an electron-positron pair, also resulting in Cherenkov light emission. This is an advantage of the water Cherenkov technique, as photons constitute a large fraction of the electromagnetic component of an air shower at ground.

HAWC improves the sensitivity for a Crab-like point spectrum by a factor of 15 in comparison to MILAGRO. The sensitivity should be also such to possibly detect gamma-ray burst emissions at high energy.

\subsubsection{Cherenkov telescopes}

Most of the experimental results on VHE photons are presently due to Imaging Atmospheric Cherenkov Telescopes (IACTs).

IACTs such as the first successful cosmic detectors, called WHIPPLE, and then the second generation instruments HEGRA and CANGAROO, and presently the third generation H.E.S.S., MAGIC and VERITAS, detect the Cherenkov photons produced in air by charged, locally superluminal particles in atmospheric showers. For reasons explained below, they have a low duty cycle (about 1000 to 1500 hours per year) and a small field-of-view (FoV), but they have a high sensitivity and a low energy threshold.

We refer to [1] for the operation principles and for the performance and the main results of these detectors.

A simplified comparison of the characteristics of the Fermi LAT satellite detector, of the IACTs and of the EAS detectors (ground-based), is given in Table 1. The sensitivities of the above described high-energy detectors are shown in Fig. 4.

The construction of the next generation IACT, called the Cherenkov Telescope Array (CTA), has already started. 
Table 1. A comparison of the characteristics of Fermi, the IACTs and of the EAS particle detector arrays. Sensitivity computed over one year for Fermi and the EAS, and over 50 hours for the IACTs.

\begin{tabular}{|l|l|l|l|}
\hline Quantity & Fermi & IACTs & EAS \\
\hline Energy range & $20 \mathrm{MeV}-200 \mathrm{GeV}$ & $100 \mathrm{GeV}-50 \mathrm{TeV}$ & $400 \mathrm{GeV}-100 \mathrm{TeV}$ \\
Energy res. & $5-10 \%$ & $15-20 \%$ & $\sim 50 \%$ \\
Duty Cycle & $80 \%$ & $15 \%$ & $>90 \%$ \\
FoV & $4 \pi / 5$ & $5^{\circ} \times 5^{\circ}$ & $4 \pi / 6$ \\
PSF & $0.1^{\circ}$ & $0.07^{\circ}$ & $0.5^{\circ}$ \\
Sensitivity & $1 \% \mathrm{Crab}(1 \mathrm{GeV})$ & $1 \% \mathrm{Crab}(0.5 \mathrm{TeV})$ & $0.5 \mathrm{Crab}(5 \mathrm{TeV})$ \\
\hline
\end{tabular}

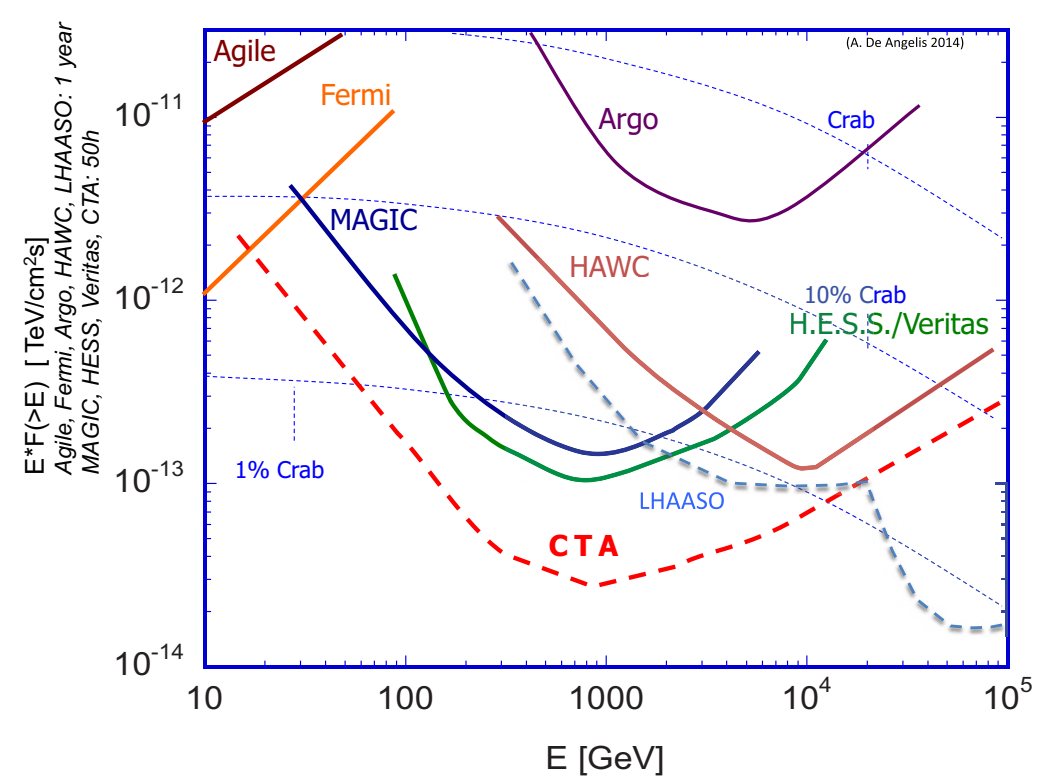

Figure 4. Sensitivities of some present and future HE gamma detectors, measured as the minimum intensity source detectable at $5 \sigma$. The performance for EAS and satellite detector is based on one year of data taking; for Cherenkov telescopes it is based on 50 hours of data. From [1].

\subsection{The near future of VHE astrophysics with Cherenkov telescopes: CTA}

The CTA [2] is a future instrument for VHE gamma astrophysics that is expected to provide an order of magnitude improvement in sensitivity over existing instruments (Fig. 4).

CTA is a robust collaboration, including more than 1000 scientists from all around the world, and large part of its funding (above 200 MEUR) is granted. CTA is included in the 2008 roadmap of the European Strategy Forum on Research Infrastructures (ESFRI). It is one of the "Magnificent Seven" experiments of the European strategy for astroparticle physics published by ASPERA, and highly ranked in the strategic plan for European astronomy of ASTRONET. In addition CTA is a recommended project for the next decade in the US National Academies of Sciences Decadal Review.

An array of tens of telescopes will detect gamma-ray induced showers over a large area on the ground, increasing the efficiency and the sensitivity, while providing a much larger number of views of each cascade. This will result in both improved angular resolution and better suppression of charged cosmic-ray background events.

In the present design scenario, CTA will be deployed in two sites. The southern hemisphere array, possibly in Paranal, will consist of three types of telescopes with different mirror sizes, in order to cover the full energy range. In the northern hemisphere array, possibly in the Canary island of La Palma, the 

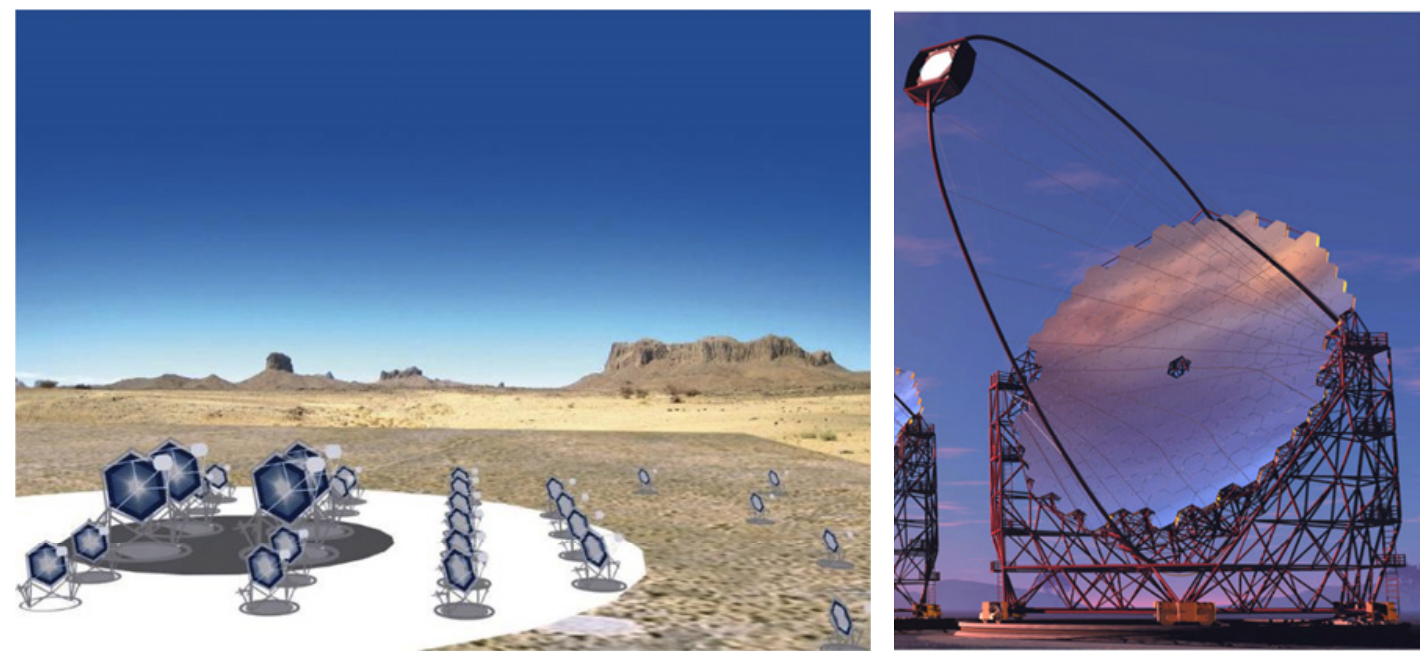

Figure 5. Left: possible layout of the CTA. Right: project of the large-size telescope (LST). Credit: CTA Collaboration.

two larger telescope types would be deployed.

- The low energy (the goal is to detect showers starting from an energy of $20 \mathrm{GeV}$ ) instrumentation will consist of four 23 meter telescopes with a FoV of about 4-5 degrees.

- The medium energy range, from around $100 \mathrm{GeV}$ to $1 \mathrm{TeV}$, will be covered by some 15-25 telescopes of the 12 meter class with a FoV of 6-8 degrees. An alternative design uses smaller reflectors (abot $9 \mathrm{~m}$ ) with a secondary optics (Schwarzschild-Couder design, [3]).

- The high energy instruments, operating above $10 \mathrm{TeV}$, will consist of a large number (of the order of 50) of small (4-6 meter diameter) telescopes with a FoV of around 10 degrees. Also in this case single-reflector designs are in competition with Schwarzschild-Couder design.

Prototyping is advanced for all these telescopes, but there is still room for technical improvement (SiPM in particular).

The southern CTA will cover about three square kilometers of land with around 60 telescopes that will monitor all the energy ranges in the center of the Milky Way's galactic plane. The northern CTA will cover one square kilometer and be composed of some 20 telescopes. These telescopes will be mostly targeted at extragalactic astronomy.

The telescopes of different sizes will be disposed in concentrical circles, the largest in the center (Fig. 5). Different modes of operation will be possible: deep field observation; pointing mode; scanning mode.

CTA is expected to surpass in performance the present IACTs around 2020.

\subsection{The near future of EAS detectors}

There is a lot of activity on the development of EAS detector, since they are relatively cheap (of the order of 30 MEUR) and we know now what is the correct size for the detection of a relevant number of sources. 


\subsubsection{HAWC+}

An upgrade of the HAWC high-energy gamma-ray observatory with a sparse array of small outrigger tanks is being investigated [4]. For showers where the core falls outside the array there are ambiguities in the reconstruction between the core position, the shower angle and the shower size or energy. An outrigger array can determine the core position for showers falling outside the main array elevating the ambiguities and making these showers well reconstructable. A gain of 3-4 in sensitivity for gammas above $10 \mathrm{TeV}$ can be obtained over what is presently achieved, and such a detector can be built in 2-3 years. Funds are already available.

\subsubsection{LHAASO}

LHAASO (Large High Altitude Air Shower Observatory) is planned to be located at about $4400 \mathrm{~m}$ asl and latitude $30^{\circ} \mathrm{N}$ in the Daochen site, China. It will consist of the following major components [5, 6]:

- $1 \mathrm{~km}^{2}$ array (LHAASO-KM2A), including 5600 scintillator detectors, with $15 \mathrm{~m}$ spacing, for electromagnetic particle detection.

- An overlapping $1 \mathrm{~km}^{2}$ array of $1200,36 \mathrm{~m}^{2}$ underground water Cherenkov tanks, with $30 \mathrm{~m}$ spacing, for muon detection (total sensitive area 40,000 $\mathrm{m}^{2}$ ).

- A close-packed, surface water Cherenkov detector facility with a total area of $90,000 \mathrm{~m}^{2}$ (LHAASO-WCDA), four times that of HAWC.

- 24 wide field-of-view air Cherenkov (and fluorescence) telescopes (LHAASO-WFCTA).

- 452 close-packed burst detectors, located near the center of the array, for detection of high energy secondary particles in the shower core region (LHAASO-SCDA).

A configuration corresponding to $25 \%$ of the final detector might be ready by 2019-2020. It is crucial that the completion of the first $25 \%$ of the detector (nominally foreseen for 2018 ) is not too much delayed with respect to the extension of HAWC, otherwise most of its scientific impact might be scooped.

\subsubsection{HiSCORE}

HiSCORE (Hundred*i Square-km Cosmic ORigin Explorer), located in the Tunka Valley near Lake Baikal at about $3200 \mathrm{~m}$ asl and latitude $51^{\circ} \mathrm{N}$, will consist of an array of wide-angle $(\Omega \sim 0.6-0.85 \mathrm{sr}$ ) light-sensitive detector stations, distributed over an area of the order of $100 \mathrm{~km}^{2}$. A HiSCORE detector station consists of four photomultiplier tubes, each equipped with a light-collecting Winston cone of $30^{\circ}$ half-opening angle [7].

The primary goal of this non-imaging Cherenkov detector is gamma-ray astronomy in the $10 \mathrm{TeV}$ to several $\mathrm{PeV}$ range. A prototype array of 9 wide-angle optical stations, spread on a $300 \times 300 \mathrm{~m}^{2}$ area, has been deployed since October 2013, and technical tests are underway. A $1 \mathrm{~km}^{2}$ engineering array has been deployed and is in commissioning.

The sensitivity of the HiSCORE standard configuration to point-sources is shown in Fig. 6. The curve has been calculated for $1000 \mathrm{~h}$ of exposure time, corresponding to roughly 1.4 years of serendipitous mode operation (see [7] for details).

HiSCORE will be possibly incorporated in TAIGA (Tunka Advanced Instrument for cosmic ray physics and Gamma Astronomy), a hybrid detector system including in particular IACTs for sensitivity to photons of lower energies. 


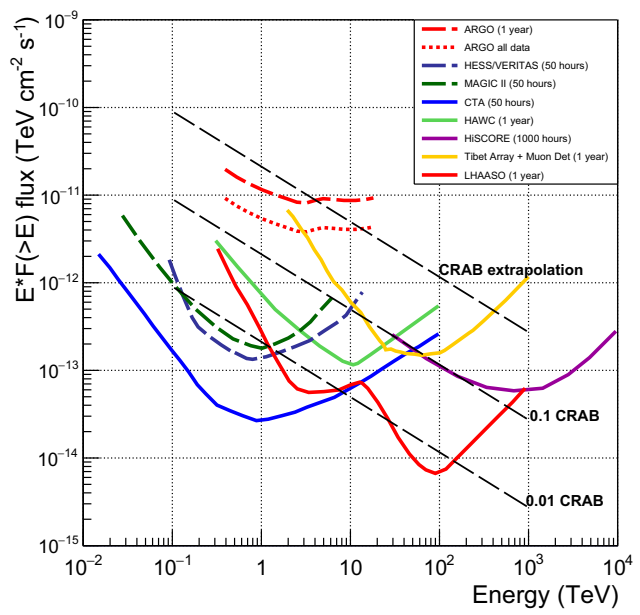

Figure 6. Sensitivity of ground-based $\gamma$-ray detectors to a Crab-like point source, not considering the attenuation of the source at large energies (from [6]).

\subsubsection{Possible EAS experiments in the Southern hemisphere}

Several proposals are being formulated now for a EAS detector at South, which would be the key to study the VHE emission from the Galaxy. HAWC has demonstrated that present EAS technology can be competitive with the technology of large-area coverage by small Cherenkov telescopes.

In particular among the proposals are:

- a Southern site for HAWC, HAWC-South, to be located in the Atacama desert [8];

- LATTES, a hybrid water Cherenkov-RPC detector to be located in Southern America [9].

\section{A view in a longer term}

Let us analyze the possible future of gamma-ray astrophysics in, say, 15 years from now.

Gamma rays can be divided in 5 different energy regions, subjectively chosen from the requirements of detection techniques and of main scientific issues. These energy regions are: (a) Till a few ( $\sim 30) \mathrm{MeV}$; (b) $\mathrm{GeV}$ : from $30 \mathrm{MeV}$ to $30 \mathrm{GeV}$ : (c) Sub-TeV: from $30 \mathrm{GeV}$ to $300 \mathrm{GeV}$; (d) $\mathrm{TeV}$ : from $300 \mathrm{GeV}$ to $30 \mathrm{TeV}$; (e) PeV: from $30 \mathrm{TeV}$ to a few PeV.

\subsection{The region till a few $\mathrm{MeV}$}

This region has important implications on the science at the $\mathrm{TeV}$ and above, since a good knowledge of the spectra in the $\mathrm{MeV}$ region can constrain the fit to the emitted spectra at high energies, thus allowing:

- to evidence additional contributions from new physics (dark matter in particular);

- to estimate cosmological absorption (due to EBL or to possible interactions with axion-like fields [10]).

On top of this, The $0.3-30 \mathrm{MeV}$ energy range is important per se, but experimentally difficult to study. It requires an efficient instrument working in the Compton regime with an excellent background subtraction, and possibly with sensitivity to the measurement of polarization. Since COMPTEL, which 
operated two decades ago, no space instrument obtained extra-solar gamma-ray data in the few $\mathrm{MeV}$ range; now we are able to build an instrument one-two orders of magnitudes more sensitive than COMTEL based on Silicon technology, state-of-the-art analog readout, and efficient data acquisition.

Several proposals (ASTROGAM [11], COMPAIR [12], ...) have been made, and convergence is likely for an experiment to be launched around 2025.

\subsection{The GeV region}

It is difficult to think for this century of an instrument for $\mathrm{GeV}$ photons improving substantially the performance of the Fermi LAT: the cost of space missions is such that the size of Fermi cannot be reasonably overcome with present technologies. New satellites in construction (like the Russian-Italian mission GAMMA400 [13] and the Chinese mission HERD [14]) improve some of the aspects of Fermi, e.g., calorimetry. For sure a satellite in the $\mathrm{GeV}$ region with sensitivity comparable with Fermi will be needed.

\subsection{The sub-TeV and TeV regions}

CTA appears to have no rivals for the gamma astrophysics in the $\mathrm{Sub}-\mathrm{TeV}$ and $\mathrm{TeV}$ (from a few $\mathrm{GeV}$ to a few $\mathrm{TeV}$ ) energy regions. These are crucial regions for fundamental physics, and for astronomy; they include also the region where sensitivity is maximum.

CTA will be probably upgraded including state-of-the art photon detection devices of higher efficiencies with respect to the present ones.

There is some room for new concepts of Cherenkov telescopes, in particular:

- a possible array of 4 or 5 very large detectors at $5000 \mathrm{~m}$ in order to boost the sensitivity around in the sub-TeV region (in particular from $20 \mathrm{GeV}$ to $100 \mathrm{GeV}$ ) [15]. This would boost the science potential in particular in sectors related to fundamental physics (dark matter, photon propagation from far sources);

- a large-FoV detector to guarantee good coverage of the sky in the TeV region [16].

\subsection{The PeV region}

Due to the opacity of the Universe to gamma rays, less than a handful of sources could be visible in the Northern sky, and less than a dozen in the Southern sky, all galactic. The experiments in the Northern hemisphere (HAWC with its likely extension to HAWC+, LHAASO, TAIGA/HiSCORE if the extension up to $10 \mathrm{~km}^{2}$ will be funded) provide an appropriate coverage of the Northern sky.

The situation in the Southern hemisphere has room for improvement. An EAS detector in the South might give substantial input with respect to the knowledge of the gamma sky, and of possible Pevatrons in the Galaxy, and outperform in this sense the SSTs of CTA. Several proposals are being formulated now, and they should join. A large detector in Southern America could compete in sensitivity with the small-size telescopes of CTA-South already at $100 \mathrm{TeV}$, offering in addition a serendipitous approach.

\section{References}

[1] A. De Angelis and M. Pimenta, Introduction to particle and astroparticle physics (Springer, Heidelberg, 2015)

[2] https://www.cta-observatory.org/

[3] V. Vassiliev et al., Astrop. Phys. 28, 10 (2007) 
[4] A. Sandoval for the HAWC Collaboration, arXiv:1509.04269, Presented ICRC2015, The Hague, The Netherlands

[5] M. Zha et al., Int. J. Mod. Phys. 10, 147 (2012)

[6] G. Di Sciascio, arXiv: 1503.05512, presented at RICAP 2014, Noto, Italy

[7] M. Tluczykont et al., Astrop. Phys. 56, 42 (2014)

[8] Michael DuVernois for the HAWC Collaboration, PoS(ICRC2015)967, Presented at ICRC2015, The Hague, The Netherlands

[9] G. Matthiae, AIP Conf. Proc. 1693 (2015) 060007 and Proc. MG14, http://w2srvg9.icra. it/upload/archivio/HE1-938MA857IO.pdf

[10] A. De Angelis, M. Roncadelli and O. Mansutti, Phys. Rev. D 76, 121301 (2007)

[11] http://astrogam.iaps.inaf.it/

[12] A. Moiseev et al., arXiv:1508.07349

[13] http://gamma400.lebedev.ru/indexeng.html

[14] http://herd.ihep.ac.cn/

[15] F. Aharonian et al., arXiv:astro-ph/0006163

[16] J. Cortina et al., http://arxiv.org/abs/1507.02532 